\title{
大气新粒子生成机制的研究
}

\author{
王志涁 胡敏* 吴志军岳玎利 \\ (北京大学环境模拟与污染控制国家重点实验室 北京大学环境科学与工程学院 北京 100871)
}

\begin{abstract}
摘要 大气中新粒子生成机制和增长特性的研究对于理解区域空气污染和全球气候变化具有十分重要的意义. 但是现 有的研究对于生成机制的认识有限. 因此, 如何通过外场观测、实验室模拟和模型计算对新粒子的生成机制进行探讨 成为大气环境科学界的研究热点和前沿. 综述了目前环境大气中新粒子生成机制和增长特性的研究方法, 总结了典型 大气环境下外场观测的研究结果, 阐述了国内外新粒子生成机制研究进展, 指出了新粒子生成研究中尚待解决的关键 科学和技术问题.
\end{abstract}

关键词 新粒子生成; 生成机制; 增长特性; 测量技术; 前体物

\section{Research on the Formation Mechanisms of New Particles in the Atmosphere}

\author{
Wang, Zhibin $\quad \mathrm{Hu}, \mathrm{Min} * \quad \mathrm{Wu}$, Zhijun $\quad$ Yue, Dingli \\ (State Key Joint Laboratory of Environmental Simulation and Pollution Control, College of Environmental Sciences and \\ Engineering, Peking University, Beijing 100871, China)
}

\begin{abstract}
New particle formation (NPF) in the atmosphere i.e. nucleation of gas phase precursors and continuing condensational growth is a crucial secondary transformation process. The freshly nucleated particles can grow into sizes where they can act as cloud condensation nuclei $(\mathrm{CCN})$ and further influence cloud and climate-relevant properties. Hence, the detailed knowledge on the formation mechanisms of new particles and their subsequent growth will help us to improve our understanding on the regional air pollution and global climate change. Recent-developed measurement techniques enable to explore atmospheric nucleation by measuring particles/clusters down to $1 \mathrm{~nm}$. NPF events have been observed in a variety of atmospheric environments. Several methods including field observation, laboratory simulations and model calculations are implemented to investigate the nucleation mechanisms and growth properties. Good agreement between particle formation rates and gaseous sulfuric acid concentrations is observed, indicating that sulfuric acid is a key compnent in the atmospheric nucleation process. In addition, the low-volatile organic vapors are found to be involved in new particle formation. However, the huge variations of the particle formation and growth rates in diverse atmospheric environments suggest that a consistent picture on formation mechanisms of new particles does not achieve. Long-term continuous measurements of NPF events have been first performed in the urban atmosphere of Beijing since 2004. Up to now, NPF events have been observed in many different environments, including coastal area, high-elevation mountain, rural site as well as urban region in China. The current nucleation mechanisms are developed based on the observation in clean environment. Hence, whether it could well explain the NPF events occurred in the polluted atmosphere with strong oxidation capacity is a key issue to be addressed in further studies. This paper reviews the measurement techniques for investigating atmospheric new particle formation and growth, summarizes the observation results in various typical environments, introduces the current understanding of the nucleation mechanisms and discusses the main scientific questions that need further researches.

Keywords new particle formation; nucleation mechanism; growth property; measurement technique; precursor
\end{abstract}

\section{1 引言}

新粒子生成(New Particle Formation, NPF)是气态污 染物氧化生成的过饱和气态前体物(例如硫酸和低挥发 性有机物等)在大气中冷凝为分子簇, 继而通过冷凝碰 并形成颗粒物的过程 ${ }^{[1 \sim 3]}$. Aitken 早在 1897 年就发现大 气中存在着新粒子生成现象 ${ }^{[4]}$, 但是受到仪器测量手段
的限制, 对这一现象的认识十分有限. 直到 20 世纪 90 年代大气中小至 $3 \mathrm{~nm}$ 的颗粒物数浓度谱分布测量仪器 的问世 ${ }^{[5,6]}$, 大气中的新粒子生成事件才被普遍地观测 和报道 ${ }^{[2,7]}$. 新粒子生成是大气中颗粒物和云凝结核的 重要来源. 最新的模型结果表明成核事件对全球颗粒物 数浓度的平均贡献远远高于一次排放 ${ }^{[8,9]}$. 新生成的颗 粒物可以后续增长至云凝结核大小, 继而通过云物理和

* E-mail: minhu@pku.edu.cn

Received December 18, 2012; published February 6, 2013.

Project supported by the National Natural Science Foundation of China (Nos. 21025728, 20977001, 21190052) and the China Ministry of Environmental Protection's Special Funds for Scientific Research on Public Welfare (No. 201009002).

项目受国家自然科学基金(Nos. 21025728, 20977001, 21190052)和国家环保公益性行业科研专项(No. 201009002)资助. 
降水过程影响地球辐射平衡和全球气候变化 ${ }^{[10]}$. 不同 大气环境下外场观测的研究结果均表明云凝结核数浓 度和颗粒物数浓度存在一定的正相关性 ${ }^{[11,12]}$, 新粒子生 成事件发生后，云凝结核的数浓度会有较大的增 长 $^{[13 \sim 17]}$, 同时全球和区域模型结果也显示新粒子生成 事件对云凝结核的数浓度贡献显著 ${ }^{[8,18 ~ 21]}$. 但是目前存 在的问题是, 研究者们对新粒子生成机制的认识有限, 因此模型的计算结果存在较大的不确定性, 继而直接影 响到对气候效应的预测 ${ }^{[22]}$.

图 1 展示了新粒子生成和增长的示意图, 主要包括 成核(Nucleation)和增长(Growth)两个过程 ${ }^{[3,23]}$. 成核是 前体物经过气态一液态一固态三相转化形成临界分子 族(critical nucleus)的过程, 在此过程中成核体系的熵和 焓都是在减小的 $(\Delta H<0$ 和 $\Delta S<0)$, 根据热力学第一定 律和第二定律, 成核过程需要克服自由能能垒 $\Delta G$. 成 核的另一个限制因素是开尔文效应(即曲率效应), 主要 是指参与成核的物种必须具有较低的饱和蒸汽压. 成核 形成的颗粒物继而通过凝结和碰并过程继续长大.

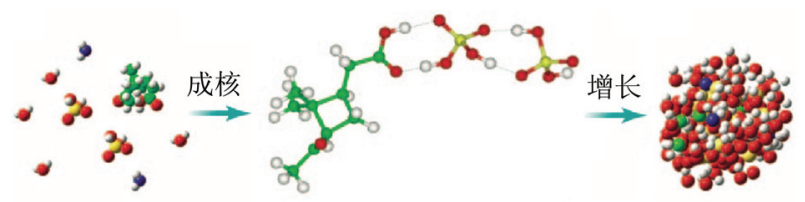

图 1 新粒子生成和增长示意图 ${ }^{[3,23]}$

Figure 1 Schematic diagram of new particle formation and growth ${ }^{[3,23]}$

在当前的研究中, 往往是根据大气中颗粒物数浓度 谱分布的变化来判断新粒子生成事件(New particle formation event) 是否发生 ${ }^{[24,25]}$, 继而根据其他参数(如前 体物浓度等)来研究其生成机制. 因此在环境大气中针 对新生成颗粒物物理化学特性和其前体物的直接测量 是研究新粒子生成机制的基础, 同时也是大气化学领域 最富挑战性的研究工作之一.

\section{2 测量技术}

外场观测是研究新粒子生成机制最为重要的手段 之一. 主要的测量参数包括新粒子气态前体物浓度、纳 米级颗粒物或正负离子的数浓度谱分布、超细颗粒物的 化学组分以及颗粒物的吸湿性和挥发性等信息. 表 1 总 结了目前外场观测中被用来研究新粒子生成机制和增 长特性的主要仪器及其测量参数.

颗粒物数浓度谱分布特征是辨识新粒子生成事件 是否发生的最基本的参数. 目前商业仪器所能测量的最 小粒径是 $3 \mathrm{~nm}^{[5,6]}$ ，最新的技术手段可观测到小至 $1 \mathrm{~nm}$ 的颗粒物和分子簇 ${ }^{[6,26 ~ 31]}$ 以及 $0.5 \mathrm{~nm}$ 的正负离子 ${ }^{[6,32]}$. 但是在大多数文献中报道的观测到的新生成颗粒物的 粒径范围主要还是集中在 $3 \sim 25 \mathrm{~nm}^{[2]}$. 大气中的气态硫 酸因为饱和蒸汽压低, 因此被认为是参与成核的关键物 种 ${ }^{[26]}$. 大气中气态硫酸的测量最早是由德国马普研究

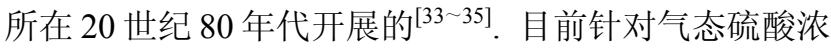
度的测量手段也在逐渐完善，常用的仪器主要是 $\mathrm{CIMS}^{[36,37]}$. 纳米级颗粒物的化学组分信息能够帮助我 们更好地认识参与成核的前体物的特性, 目前包括 TD-CIMS 和 AMS 等多种仪器都被运用于外场观测之 中, 但是相对于新粒子的研究, 这些仪器测量的粒径范 围仍旧偏大，同时不同仪器之间的测量结果存在着较大 的不确定性 ${ }^{[38]}$. 目前新粒子研究的仪器测量手段在不 断进步，但是对成核机制的实测研究仍旧存在着很大的 局限性.

\section{3 新粒子生成事件的观测和成核机制研究进展}

\section{1 新粒子生成事件的观测}

随着测量仪器的发展, 从 20 世纪 90 年代至今, 新 粒子生成事件在全球范围内不同大气环境下被普遍观 察到, 同时发现其有区域性等特征 ${ }^{[2,7,56 ~ 59]}$. 国际上长期

表 1 外场观测研究新粒子生成的仪器及主要参数

Table 1 List of the instruments and measurement parameters in field observation

\begin{tabular}{|c|c|c|c|c|}
\hline No. & Instrument & Measurement parameter & Size range/nm & Ref. \\
\hline 1 & ID-CIMS & Sulfuric acid concentration & - & {$[33 \sim 36]$} \\
\hline 2 & PTR-MS & Volatile Organic Compounds (VOCs) concentration & - & {$[39 \sim 41]$} \\
\hline 3 & SMPS/DMPS & Particle number size distribution & $\approx 3$ & {$[5,42]$} \\
\hline 4 & PSM & Particle number concentration & $\approx 1$ & {$[6,43]$} \\
\hline 5 & AIS/BSMA & Ion size distribution & $\approx 0.5$ & {$[29,44]$} \\
\hline 6 & Cluster CIMS & Neutral molecular clusters & $\approx 1$ & {$[28,30]$} \\
\hline 7 & TD-CIMS & Size-resolved chemical composition & $6 \sim 20$ & {$[45 \sim 48]$} \\
\hline 8 & NAMS & Size-resolved chemical composition & $\approx 25$ & {$[49 \sim 52]$} \\
\hline 9 & HR-ToF-AMS & Size-resolved chemical composition & $30 \sim 600$ & {$[38,53]$} \\
\hline 10 & VTDMA/HTDMA & Hygroscopicity/volatility & - & {$[54,55]$} \\
\hline
\end{tabular}

1 2: Gaseous precursors; 3 6: Particle/Ion/cluster size distributions; 7 9: Nano-size chemical compositions; 10: Other properties; ID-CIMS, Ion Drift-Chemical Ionization Mass Spectrometer; PTR-MS, Proton Transfer Reaction Mass Spectrometry; SMPS, Scanning Mobility Particle Sizer; DMPS, Differential Mobility Particle Sizer; PSM, Particle size magnifier; AIS, Air Ion Spectrometer; BSMA, Balanced Scanning Mobility Analyzer; Cluster CIMS, Cluster Chemical Ionization Mass Spectrometer; TD-CIMS, Thermal Desorption-Chemical Ionization Mass Spectrometer; NAMS, Nano Aerosol Mass Spectrometer; HR-ToF-AMS, High Resolution Time of Flight Aerosol Mass Spectrometer; VTDMA, Volatility Tandem Differential Mobility Analyzer; HTDMA, Hygroscopic Tandem Differential Mobility Analyzer. 
(1 年以上)开展颗粒物数谱分布研究的地点如图 2 所示, 这些观测跨越了多种典型的环境条件，例如城市、海洋、 森林、高山、乡村和极地等. 但是这些研究主要集中在 欧洲和美国等发达国家, 在发展中国家的长期研究较 少.

生成速率(Formation Rate, FR)和增长速率(Growth Rate, GR) 是表征新粒子生成和增长的两个重要参数 ${ }^{[6]}$. 生成速率表示成核过程中新生成颗粒物数浓度随时间 的变化, 增长速率表示核模态颗粒物的中值粒径随时间 的变化. Kulmala 等 ${ }^{[2]}$ 基于 100 多次外场观测的结果发现 新粒子的生成速率 $\left(J_{3}\right)$ 具有较强的地域依赖性, 一般地 区的生成速率大约在 $0.01 \sim 10 \mathrm{~cm}^{-3} \cdot \mathrm{s}^{-1}$ 之间, 城市地区 则明显较高 $\left(100 \mathrm{~cm}^{-3} \cdot \mathrm{s}^{-1}\right)$, 而在海岸地区和工业区甚至 能够达到 $10^{4} \sim 10^{5} \mathrm{~cm}^{-3} \cdot \mathrm{s}^{-1}$. 但是增长速率并未呈现出 较大的差异, 一般在 $1 \sim 20 \mathrm{~nm} / \mathrm{h}$ 之间. 一些典型大气环 境下的新粒子生成速率和增长速率如图 3 所示, 虽然大 部分地区的生成速率和增长速率并没有呈现出显著差 异, 但是发展中国家整体污染情况比较严重的超大型城 市(如新德里和北京)新粒子生成时的可凝结气态前体物 浓度、可凝结气态前体物产生速率和凝结汇较高 ${ }^{[80,81]}$.

\section{2 新粒子生成机制的研究进展}

针对新粒子生成机制的研究很早已经开始 ${ }^{[95]}$, 至

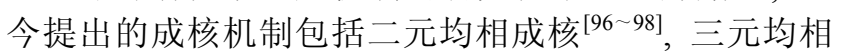
成核 ${ }^{[99 \sim 102]}$, 离子诱导成核 ${ }^{[9,22,103,104]}$ 和碘参与成 核 ${ }^{855,105 ~ 108]}$ 等. 但是这些成核理论都有各自的适用范围, 例如二元成核理论只有在满足低温、高湿、大气中已存 在的颗粒物比较少、气态硫酸浓度比较高的特定环境下 才能发生 ${ }^{[109]}$, 它不能解释生成速率较高的海岸和城市 地区的成核现象. 而针对碘参与成核的研究主要集中在
海岸环境下. 因此目前并没有一个成熟的成核机理来代 表全球的情况.

由于气态硫酸被证明是参与成核的关键物种，因此 当前的研究主要集中于生成速率和气态硫酸浓度指数 之间的关系. 同时为了满足模型参数化的需要, 生成机 制的表达方式可以简单地描述为 $J=\left[\mathrm{H}_{2} \mathrm{SO}_{4}\right]^{n}, n$ 可以理 解为分子族中所包含的硫酸个数 ${ }^{[110]}$. 在国外干洁的大 气环境下的观测结果显示 $n$ 值介于 $1 \sim 2$ 之间, 表明成核 过程可能是活化成核和动力学成核共同作用的结 果 $^{[71,90,111 ~ 114]}$, 但是在污染环境例如北京城区的研究发 现 $n$ 值的范围较大 ${ }^{[115]}$. 但最新的研究结果表明动力学 成核理论同外场观测结果的吻合程度远优于传统热力 学理论 ${ }^{[116,117]}$, 同时在污染严重的环境中(如北京, 墨西 哥)动力学成核的成核系数比干洁地区要高出 $1 \sim 2$ 个数 量级，表明在污染严重的城市地区成核物种和干洁地区 有很大差别 ${ }^{[111,115,116,118 ~ 122]}$. 同时大气中的气态硫酸浓 度对新生成颗粒物增长速率的解释不足 $20 \%$ 也暗示新 粒子生成过程中会有其他物种的参与 ${ }^{[32,45,123 ~ 127]}$.

大气中氨的存在会大大降低气态硫酸分子的表面 蒸汽压, 这样氨可能与大气中的硫酸和水均相成核. 外 场观测和实验室模拟都表明氨在新粒子的增长中有重 要作用 ${ }^{[46,47,54,128 ~ 131]}$. 由于挥发性有机物在大气中的浓 度很高, 其氧化产物的挥发性较低, 也被认为可能参与 到新粒子生成过程 ${ }^{[132]}$. 包括有机酸、有机胺在内的多种 有机物都被外场观测和实验室研究证明在新粒子生成 过程中具有重要的作用 ${ }^{[45,48,130,133 ~ 142]}$. 同时和无机氨相 比, 有机胺和硫酸分子之间可以结合形成更为稳定的分 子簇，对新生成颗粒物数浓度增长的影响更为显

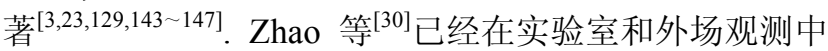

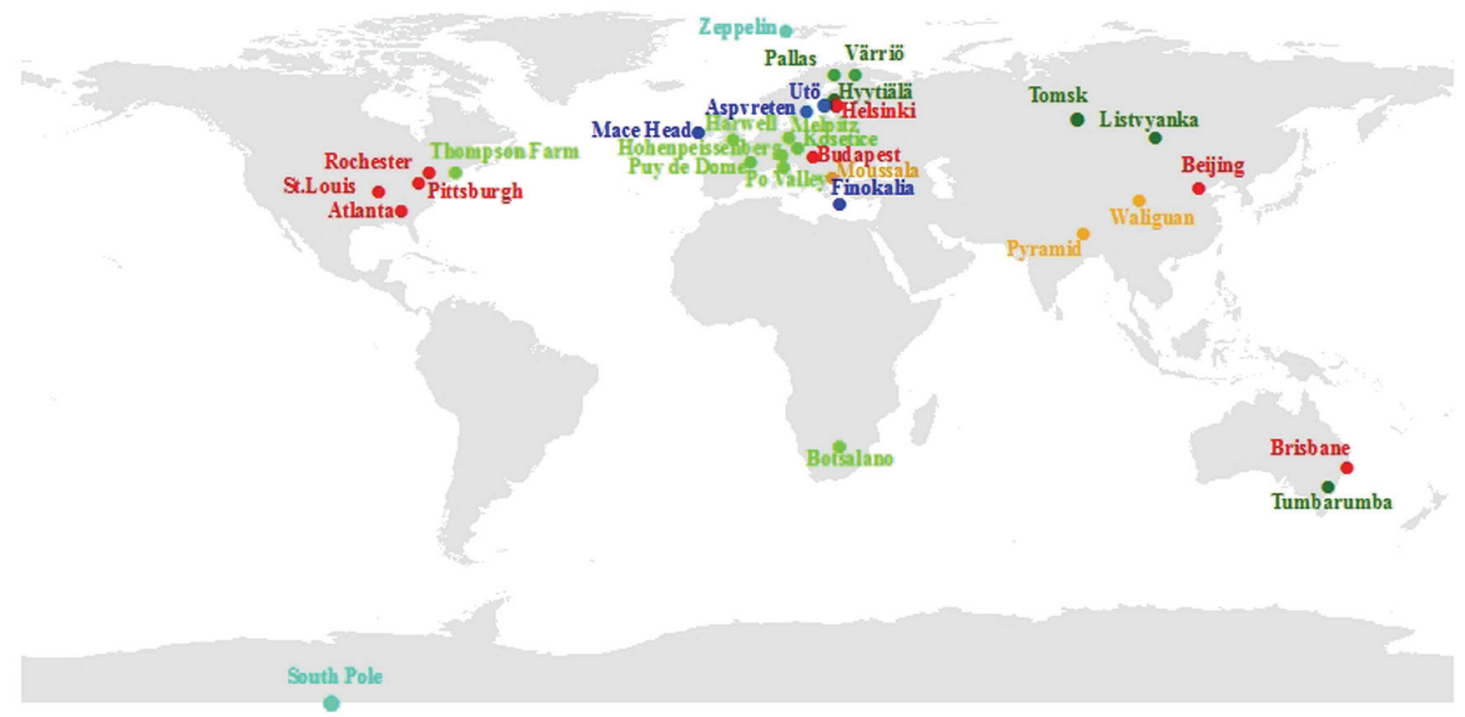

图 2 文献中报道的全球颗粒物数谱分布长期(1 年以上)观测站点; 其中红色代表城市站点; 蓝色代表海洋站点; 深绿代表森林站点; 浅绿代表郊 区站点; 黄色代表高山站点; 青色代表极地站点 ${ }^{[2,24,25,60 ~ 79]}$

Figure 2 Locations of the long term observations ( $>1$ year) of particle number size distributions in the world. The observation sites in diverse atmospheric environments are labeled as colored dots. Red represents urban site, blue denotes coastal environment, dark green indicates forest site, green describes rural site, mountain site and polar site are marked as yellow and cyan, respectively ${ }^{[2,24,25,60 \rightarrow 79]}$ 


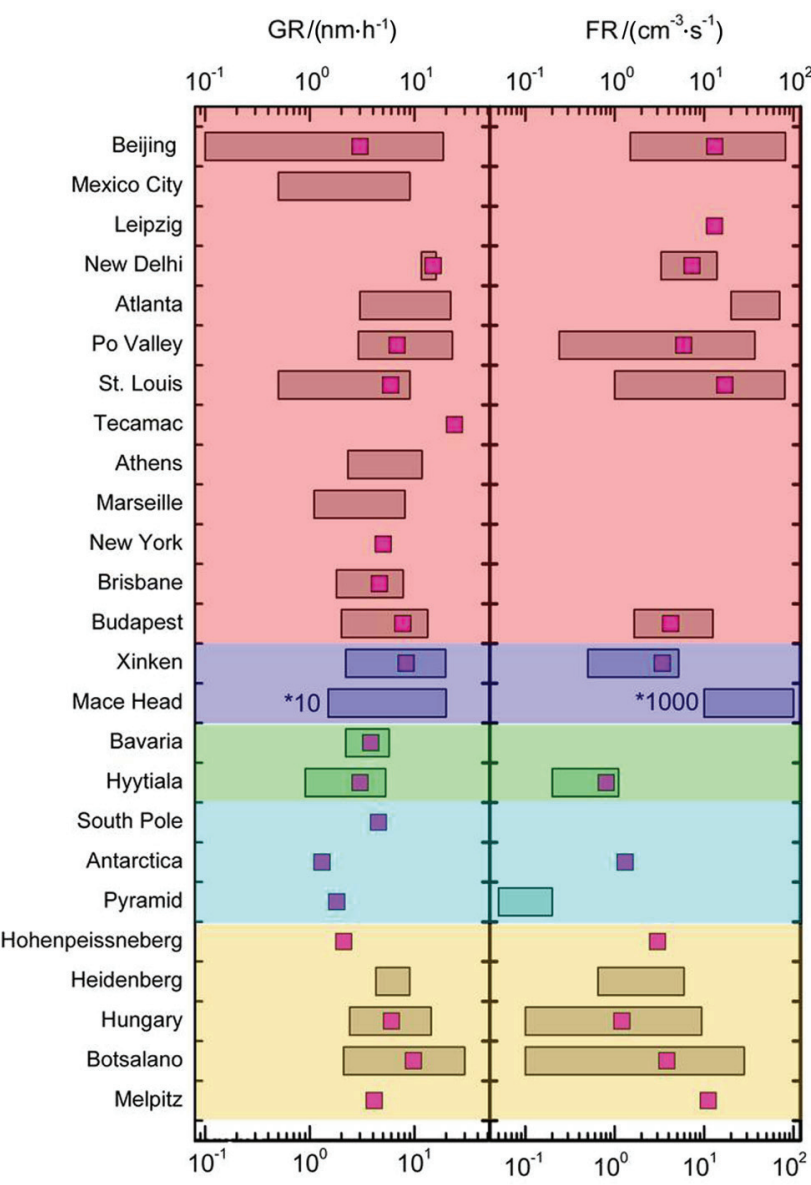

图 3 不同环境下新粒子的生成速率和增长速率 $[1,2,24,25,45,61,65,66,78,81 \sim 94]$, 红色背景为城市站点, 紫色为海岸站点, 绿色为森林站点, 青色为偏 远地区, 黄色为乡村站点

Figure 3 The observed formation rate (FR) and growth rate (GR) in diverse atmospheric environments. Red background represents urban site, purple decribes coastal environment, green indicates forest site, remote and rural sites are marked as cyan and yellow, respectively. The pink dot denotes the mean value

用 Cluster-CIMS 检测到了含有 4 个硫酸分子和 1 个有机 胺的中性分子簇. 低挥发性有机物在大气中种类繁多, 大气中氧化产物的结构更加复杂多变. 目前还不能确定 究竟是哪一种或哪一类型的有机物参与到成核过程当 中. 同时也有相关研究表明有机物会对新粒子生成起到 抑制作用 ${ }^{[148,149]}$. 目前国际上普遍认为有机物对新生成 颗粒物的增长具有非常重要的作用 ${ }^{[38,150,151]}$, 但是其究 竟是否参与成核过程, 并在当中起什么作用仍有待于进 一步研究.

综上所述, 虽然目前提出的多种成核理论都在一定 大气环境下被证实, 但是针对新粒子的成核机理仍存在 较大的争议. 气态硫酸是新粒子生成的关键前体物, 而 各种有机物也被证实可能会参与到新粒子生成过程当 中. 但是以上的研究工作主要集中在欧美等发达国家大 气环境较为干洁的地区, 在这样的背景下所提出的生成 机制能否解释我国高污染强氧化性背景下发生的新粒
子生成现象仍有待研究.

\section{4 中国的研究进展}

相对于西方发达国家，我国对颗粒物数谱分布和新 粒子生成事件的研究起步较晚. 2004 年 3 月, 在北京大 学城市大气环境定位观测站采用双差分电迁移颗粒物 粒径谱仪首次观测到新粒子生成事件的发生 ${ }^{[152]}$. 同时 近年来在城市 ${ }^{[24,153 \sim 158]}$, 乡村 ${ }^{[74,159 \sim 161]}$, 海岸 ${ }^{[86,162,163]}$, 高山 ${ }^{[164,165]}$ 等大气环境下也都观测到了新粒子生成事件 的发生(表 2), 但是长期的颗粒物数谱分布的测量只在 北京城区 ${ }^{[69]}$ ，上甸子 ${ }^{[74]}$ 和瓦里关 ${ }^{[70]}$ 有过报道.

国内针对新粒子生成规律和机制的研究主要在北 京展开. $\mathrm{Wu}$ 等 ${ }^{[24]}$ 在一年观测数据的基础上给出了北京 城市大气新粒子生成的判定标准和分类. 北京新粒子生 成事件发生频率为春冬最高, 秋季次之, 夏季最低. $2004 \sim 2008$ 年季节平均爆发频率为 $2 \% \sim 40 \%$, 总体平 均为 $21 \%$. 北京新粒子生成主要特征参数季节变化如图 4 所示. 总体而言新粒子的生成速率在冬春较高, 而增 长速率, 可凝结气态前体物浓度及其产生速率在夏天最 高. 在对北京新粒子生成规律和特征参数基本把握的基 础上, Yue 等 ${ }^{[157]}$ 根据气态硫酸凝结及其中和对增长速率 的贡献将北京新粒子生成事件分为富硫型和贫硫型两 种增长类型. 而在最新的研究中对重要前体物气态硫酸 在新粒子生成过程中的作用也进行了探讨 ${ }^{[115]}$. 对珠三 角地区新粒子生成事件特征的研究表明相比于北京地 区其增长速率和凝结汇较高, 三元成核是该地区新粒子 生成事件中可能的核化机理, 新生成颗粒物的后续增长 还需要其他低挥发性气态前体物的参与 ${ }^{[163,166]}$.

总体而言, 国内对新粒子生成事件的研究存在以下 不足: (1)所测量的大气颗粒物的粒径较大, 占颗粒物数 浓度主体的超细粒子, 尤其是粒径小于 $20 \mathrm{~nm}$ 的核模态 颗粒物较少被测量, 难以识别新粒子生成事件; (2)仅有 北京大学城市大气环境定位观测站, 上甸子和瓦里关区 域背景站等少数长期连续的观测结果, 无法洞悉国内新 粒子生成事件的特征和变化规律; (3)鲜有同期观测的新 粒子前体物如气态硫酸的浓度与 $20 \mathrm{~nm}$ 以下颗粒物化学 组成的信息, 新粒子生成机制与超细颗粒物化学组成综 合研究不够深入, 难以对新粒子生成机制与增长特性进 行研究.

\section{5 结论与展望}

新粒子生成是低挥发性可凝结气态前体物从气态 向颗粒态转化的主要形式之一, 是全球颗粒物和云凝结 核的重要来源. 但是由于对成核机制认识的不足, 也是 全球和区域气候模型中不确定性的重要来源. 虽然国际 上在不同典型大气环境下都观测到了高频率发生的新 粒子生成事件, 但是由于仪器检测手段的限制, 针对其 
表 2 我国不同环境下新粒子的生成速率和增长速率 ${ }^{a}$

Table 2 The observed formation rate (FR) and growth rate (GR) in diverse atmospheric environments of China

\begin{tabular}{|c|c|c|c|c|c|}
\hline Site & Air mass type & Time period & $\mathrm{FR} /\left(\mathrm{cm}^{-3} \cdot \mathrm{s}^{-1}\right)$ & $\mathrm{GR} /\left(\mathrm{nm} \bullet \mathrm{h}^{-1}\right)$ & Ref. \\
\hline Beijing & Urban & March 2004 February 2005 & $3.3 \sim 81.4\left(J_{3}\right)$ & $0.1 \sim 11.2$ & {$[24]$} \\
\hline Shanghai & Urban & October 2008 February 2009 & $0.2 \sim 0.5\left(J_{10}\right)$ & $3.3 \sim 5.5$ & {$[153]$} \\
\hline Lanzhou & Suburban & June $\sim$ July 2006 & $1.8 \sim 7.1\left(J_{10}\right)$ & $1.3 \sim 17.0$ & {$[155]$} \\
\hline Taicang & Suburban & May 2005 & $1.2 \sim 2.5\left(J_{10}\right)$ & $3.6 \sim 7.4$ & [156] \\
\hline Nanjing & Rural & November $2011 \sim$ March 2012 & $1.1\left(J_{6}\right)^{a}$ & $8.5^{a}$ & {$[161]$} \\
\hline Shangdianzi & Rural/Regional & March 2008 August 2009 & $0.7 \sim 72.7\left(J_{3}\right)$ & $0.3 \sim 14.5$ & {$[74]$} \\
\hline Xinken & Rural/Coastal & October $\sim$ November 2004 & $0.5 \sim 5.2\left(J_{3}\right)$ & $2.2 \sim 19.8$ & {$[86]$} \\
\hline Yellow Sea & Marine & March and May 2005 & - & $3.4^{a}$ & {$[162]$} \\
\hline Hongkong & Mountain & 25 October $\sim 29$ November 2010 & $1.0 \sim 10.2\left(J_{5.5}\right)$ & $1.5 \sim 8.4$ & {$[165]$} \\
\hline
\end{tabular}

${ }^{a}$ Indicates mean value.

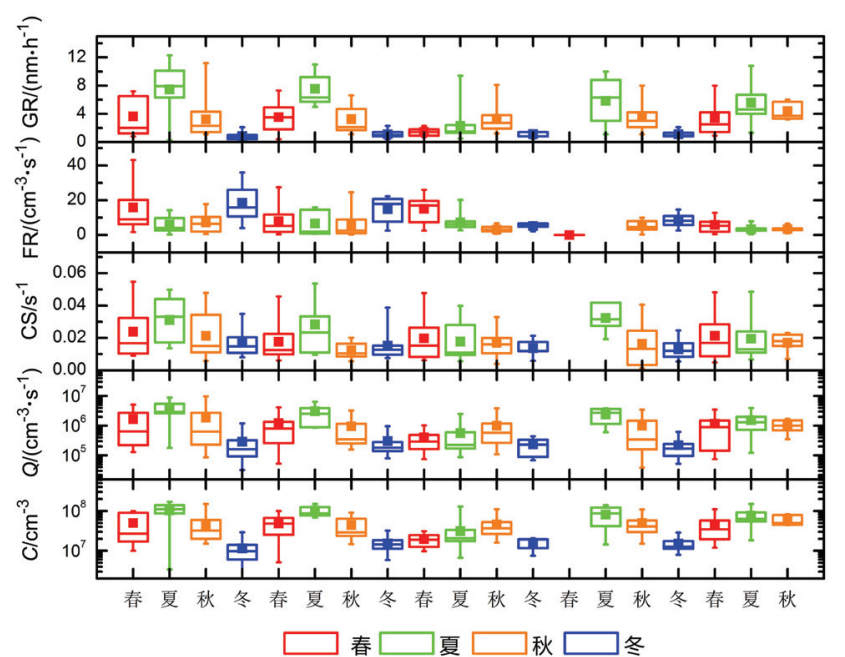

图 4 北京 2004 年至 2008 年新粒子生成特征参数季节变化(左起为 2004 年春). 盒子图的上下边界分别表示 75 和 25 分位数, 中间横线表 示中值, 上下边缘表示 95 和 5 分位数, 方块表示均值. GR: 增长速率; FR: 生成速率; $\mathrm{CS}$ : 凝结汇; $Q$ : 可凝结气态前体物分子产生速率; $C$ : 可凝结气态前体物浓度. 春季: 3 月 5 月, 红色; 夏季: 6 月 8 月, 绿 色; 秋季: 9 月 11 月, 桔黄色; 冬季: 12 月～次年 2 月; 蓝色

Figure 4 The seasonal variations of basic parameters of new particle formation in the urban environment of Beijing from 2004 to 2008 . The upper and lower boundaries of boxes indicate the 75th and 25th percentiles; the line within the box marks the median; the whiskers above and below boxes indicate the 95th and 5th percentiles; and square symbols represent the means. GR: growth rate; FR: formation rate; CS: condensation sink; $Q$ : source rate; $C$ : vapor concentration. Spring: March $\sim$ May, red; Summer: June $\sim$ August, green; Autumn: September $~$ November, orange; Winter: December $\sim$ February (next year), blue

生成机制的研究仍旧存在较大的争议. 目前讨论的生成 机制都是基于在大气环境较为干洁的地区的研究所提 出的, 在污染严重的城市地区, 由于大气成分比较复杂, 各物种在成核中的作用是否和干洁地区相同是本领域 讨论的热点. 基于以上讨论, 笔者认为新粒子生成领域 未来的研究方向主要是:

(1) 完善新粒子生成的重要前体物气态硫酸的测量 和模拟方法. 大气中的气态硫酸是新粒子生成的关键物 种, 对其浓度的测量对新粒子生成机制的研究至关重 要. 同时在模型中针对气态硫酸生成和去除机制的模拟 有助于更好地认识其在大气中的化学过程.

(2) 开展不同大气环境下(污染地区和干洁地区)颗
粒物数谱分布和前体物浓度的长期观测. 研究表明较为 干洁的背景地区和较为污染的城市地区新粒子的生成 机制和增长特性差异较大, 不能在模型中用单一的生成 机制来代表整个区域甚至全球的情况. 因此应选择典型 的大气环境(污染和干洁), 同时开展颗粒物数谱分布和 气态前体物浓度等参数的测量, 通过对比研究得到具有 普适性的生成机制.

（3）探讨有机物在成核过程中的贡献和作用. 虽然 部分外场观测和实验室模拟研究结果表明有机物可能 会作为前体物参与成核过程当中, 但是针对具体有机物 的物种存在较大的争议. 因此在接下来的研究中可以利 用烟雾箱对可能参与成核的有机物物种进行辨识, 通过 大量条件实验来确定新粒子可能的生成机制和其表达 式中的关键参数, 并对外场观测实验的设计提供指导.

(4) 评估新粒子生成对气候变化的影响. 模型中新 粒子成核机制的参数化设定存在较大的不确定性. 因此 在大量外场观测和实验室模拟的基础上，对具有普适性 的生成机制参数化，从而降低新粒子生成对区域和全球 气候模型不确定性的影响, 提高研究者对气候变化预测 的精确度.

\section{作者简介}

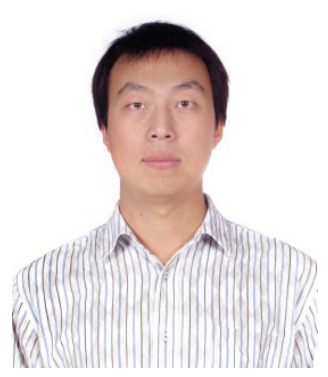

王志彬, 博士. 2012 年毕业于北京大学环境科学与工程学 院. 毕业后至今在德国莱布尼茨对流层研究所进行博士后研 究. 主要研究方向包括颗粒物物理化学特征的演变, 侧重于 大气新粒子生成机制与增长特性及其环境效应等. 参加的课 题包括 “十一五” 863 项目课题 “珠江三角洲区域大气复合污 染立体监测网络”、重大国际合作项目“北京细、超细颗粒 物数浓度和化学成分谱分布及吸水性研究”、国家自然科学 
基金面上项目和环保部公益项目等。第一作者发表 SCI 文章 2 篇.

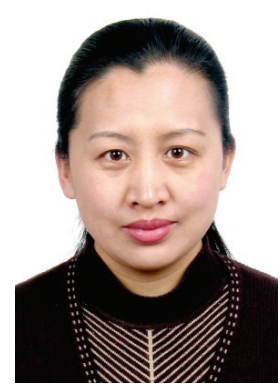

胡敏, 博士. 北京大学教授, 博士生导师. 1993 年毕业于 北京大学环境科学中心. 多年来从事细颗粒物化学行为、来源 解析及环境影响等关键科学问题的研究. 建立了颗粒物物理 化学特性分析与模式模拟方法、建立并开展了城市大气环境 定位观测站长期观测、主持和参与了多次大型观测实验, 推动 了我国大气监测和化学分析水平的全面提升. 分别于 2004 年 和 2011 年获得教育部 “新世纪优秀人才支持计划” 和“杰出 青年基金” 资助. 2011 年荣获“中国青年女科学家”. 2007 年 以来参加了包括国家 863,973 和自然科学基金等课题 22 项. 获国家科技进步二等奖 1 项(排名第 2)、部级科技进步一等奖 2 项(排名第 2 和第 9). 发表 SCI 文章 100 篇.

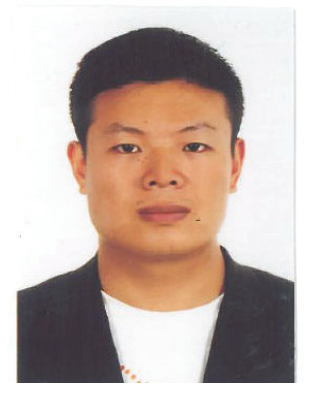

吴志军, 博士. 2007 年毕业于北京大学环境学院. 毕业后 至今在德国莱布尼茨对流层研究所进行博士后研究. 主要研 究兴趣包括大气环境中新粒子生成与增长机理; 颗粒物挥发、 吸湿性与化学测量方法结合研究有机气溶胶转化机制; 海洋 气溶胶有机组份来源转化及其气候效应. 目前主要参与的项 目有德国联邦政府资助的 “海洋与大气间能源与物质交换” 和中德科学中心资助的 “一种基于实地、雷达和卫星协同测 量研究气溶胶污染对区域尺度健康影响的新手段”. 近五年在 国际杂志上发表学术论文 33 篇.

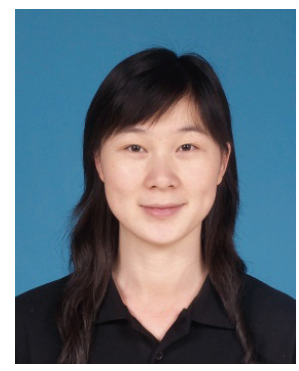

岳玎利, 博士, 高级工程师. 2010 年博士毕业于北京大学 环境科学与工程学院. 毕业后至今在广东省环境监测中心工 作. 主要研究方向为大气环境化学, 开展新粒子生成机制与 增长特性等气溶胶理化特性的相关研究. 目前负责中国广东 大气超级站业务化运行与数据挖掘. 参加的课题包括 “十一
五” 863 项目课题 “珠江三角洲区域大气复合污染立体监测网 络”、重大国际合作项目 “北京细、超细颗粒物数浓度和化 学成分谱分布及吸水性研究”、国家自然科学基金面上项目、 广东省自然科学基金重点项目和环保部公益项目等。第一作 者发表 SCI 文章 5 篇.

\section{References}

[1] Birmili, W.; Wiedensohler, A. Geophys. Res. Lett. 2000, 27(20), 3325.

[2] Kulmala, M.; Vehkamäki, H.; Petäjä, T.; Dal Maso, M.; Lauri, A.; Kerminen, V. M.; Birmili, W.; McMurry, P. H. J. Aerosol Sci. 2004 35(2), 143.

[3] Zhang, R.; Khalizov, A.; Wang, L.; Hu, M.; Xu, W. Chem. Rev. 2012, 112(3), 1957.

[4] Aitken, J. A. Trans. Roy. Soc. 1897, XXXIX, 15.

[5] McMurry, P. H. Atmos. Environ. 2000, 34(12-14), 1959.

[6] Kulmala, M.; Petäjä, T.; Nieminen, T.; Sipilä, M.; Manninen, H. E.; Lehtipalo, K.; Dal Maso, M.; Aalto, P. P.; Junninen, H.; Paasonen, P.; Riipinen, I.; Lehtinen, K. E. J.; Laaksonen, A.; Kerminen, V.-M. Nat. Protocols 2012, 7(9), 1651

[7] Holmes, N. S. Atmos. Environ. 2007, 41(10), 2183.

[8] Merikanto, J.; Spracklen, D. V.; Mann, G. W.; Pickering, S. J.; Carslaw, K. S. Atmos. Chem. Phys. 2009, 9(21), 8601.

[9] Yu, F. Q.; Wang, Z.; Luo, G.; Turco, R. Atmos. Chem. Phys. 2008, 8(9), 2537.

[10] Kerminen, V. M.; Lihavainen, H.; Komppula, M.; Viisanen, Y.; Kulmala, M. Geophys. Res. Lett. 2005, 32(14), L14803.

[11] Ramanathan, V.; Crutzen, P. J.; Kiehl, J. T.; Rosenfeld, D. Science 2001, 294(5549), 2119.

[12] Laaksonen, A.; Hamed, A.; Joutsensaari, J.; Hiltunen, L.; Cavalli, F.; Junkermann, W.; Asmi, A.; Fuzzi, S.; Facchini, M. C. Geophys. Res. Lett. 2005, 32(6), L06812.

[13] O'Dowd, C. D. J. Geophys. Res. 2001, 106(D2), 1545.

[14] Lihavainen, H.; Kerminen, V. M.; Komppula, M.; Hatakka, J.; Aaltonen, V.; Kulmala, M.; Viisanen, Y. J. Geophys. Res. 2003, 108(D24), 4782.

[15] Kuwata, M.; Kondo, Y.; Miyazaki, Y.; Komazaki, Y.; Kim, J. H.; Yum, S. S.; Tanimoto, H.; Matsueda, H. Atmos. Chem. Phys. 2008, 8(11), 2933.

[16] Yue, D. L.; Hu, M.; Zhang, R. Y.; Wu, Z. J.; Su, H.; Wang, Z. B.; Peng, J. F.; He, L. Y.; Huang, X. F.; Gong, Y. G.; Wiedensohler, A. Atmos. Environ. 2011, 45(33), 6070.

[17] Kuang, C.; McMurry, P. H.; McCormick, A. V. Geophys. Res. Lett. 2009, 36(9), L09822.

[18] Spracklen, D. V.; Carslaw, K. S.; Kulmala, M.; Kerminen, V.-M.; Sihto, S.-L.; Riipinen, I.; Merikanto, J.; Mann, G. W.; Chipperfield, M. P.; Wiedensohler, A.; Birmili, W.; Lihavainen, H. Geophys. Res. Lett. 2008, 35(6), L06808.

[19] Pierce, J. R.; Adams, P. J. Atmos. Chem. Phys. 2009, 9(4), 1339.

[20] Pierce, J. R.; Leaitch, W. R.; Liggio, J.; Westervelt, D. M.; Wainwright, C. D.; Abbatt, J. P. D.; Ahlm, L.; Al-Basheer, W.; Cziczo, D. J.; Hayden, K. L.; Lee, A. K. Y.; Li, S. M.; Russell, L. M.; Sjostedt, S. J.; Strawbridge, K. B.; Travis, M.; Vlasenko, A.; Wentzell, J. J. B.; Wiebe, H. A.; Wong, J. P. S.; Macdonald, A. M. Atmos. Chem. Phys. 2012, 12(7), 3147.

[21] Yu, F. Q.; Luo, G. Atmos. Chem. Phys. 2009, 9(20), 7691.

[22] Yu, F.; Luo, G.; Bates, T. S.; Anderson, B.; Clarke, A.; Kapustin, V.; Yantosca, R. M.; Wang, Y.; Wu, S. J. Geophys. Res.-Atmos. 2010, 115 (D17), D17205.

[23] Zhang, R. Y. Science 2010, 328(5984), 1366.

[24] Wu, Z. J.; Hu, M.; Liu, S.; Wehner, B.; Bauer, S.; Ma ßling, A.; Wiedensohler, A.; Petäjä, T.; Dal Maso, M.; Kulmala, M. $J$. Geophys. Res. 2007, 112(D9), D09209.

[25] Dal Maso, M.; Kulmala, M.; Riipinen, I.; Wagner, R.; Hussein, T.; Aalto, P. P.; Lehtinen, K. E. J. Boreal Environ. Res. 2005, 10(5), 323.

[26] Sipila, M.; Berndt, T.; Petaja, T.; Brus, D.; Vanhanen, J.; Stratmann, F.; Patokoski, J.; Mauldin, R. L.; Hyvarinen, A. P.; Lihavainen, H.; Kulmala, M. Science 2010, 327(5970), 1243.

[27] Jiang, J.; Zhao, J.; Chen, M.; Eisele, F. L.; Scheckman, J.; Williams, B. J.; Kuang, C.; McMurry, P. H. Aerosol Sci. Technol. 2011, 45(4), ii-v. 
[28] Zhao, J.; Eisele, F. L.; Titcombe, M.; Kuang, C.; McMurry, P. H. J. Geophys. Res. 2010, 115(D8), D08205.

[29] Kulmala, M.; Riipinen, I.; Sipilä, M.; Manninen, H. E.; Petäjä, T.; Junninen, H.; Maso, M. D.; Mordas, G.; Mirme, A.; Vana, M.; Hirsikko, A.; Laakso, L.; Harrison, R. M.; Hanson, I.; Leung, C.; Lehtinen, K. E. J.; Kerminen, V.-M. Science 2007, $318(5847), 89$.

[30] Zhao, J.; Smith, J. N.; Eisele, F. L.; Chen, M.; Kuang, C.; McMurry, P. H. Atmos. Chem. Phys. 2011, 11(21), 10823.

[31] Zhao, J.; Khalizov, A. F.; Zhang, R.; McGraw, R. J. Phys. Chem. A 2009, 113(4), 680.

[32] Kulmala, M.; Kerminen, V.-M. Atmos. Res. 2008, 90(2-4), 132.

[33] Arnold, F.; Fabian, R. Nature 1980, 283(5742), 55.

[34] Arnold, F.; Viggiano, A. A.; Schlager, H. Nature 1982, 297(5865), 371.

[35] Arnold, F.; Buhrke, T. Nature 1983, 301(5898), 293.

[36] Fortner, E. C.; Zhao, J.; Zhang, R. Y. Anal. Chem. 2004, 76(18), 5436.

[37] Zheng, J.; Khalizov, A.; Wang, L.; Zhang, R. Anal. Chem. 2010, 82(17), 7302.

[38] Riipinen, I.; Yli-Juuti, T.; Pierce, J. R.; Petäjä, T.; Worsnop, D. R.; Kulmala, M.; Donahue, N. M. Nat. Geosci. 2012, 5(7), 453.

[39] Sellegri, K.; Hanke, M.; Umann, B.; Arnold, F.; Kulmala, M. Atmos. Chem. Phys. 2005, 5(2), 373.

[40] Taipale, R.; Ruuskanen, T. M.; Rinne, J.; Kajos, M. K.; Hakola, H.; Pohja, T.; Kulmala, M. Atmos. Chem. Phys. 2008, 8(22), 6681.

[41] Hao, L. Q.; Yli-Pirilä, P.; Tiitta, P.; Romakkaniemi, S.; Vaattovaara, P.; Kajos, M. K.; Rinne, J.; Heijari, J.; Kortelainen, A.; Miettinen, P.; Kroll, J. H.; Holopainen, J. K.; Smith, J. N.; Joutsensaari, J.; Kulmala, M.; Worsnop, D. R.; Laaksonen, A. Atmos. Chem. Phys. 2009, $9(20), 8121$.

[42] Wiedensohler, A.; Birmili, W.; Nowak, A.; Sonntag, A.; Weinhold, K.; Merkel, M.; Wehner, B.; Tuch, T.; Pfeifer, S.; Fiebig, M.; Fjäraa, A. M.; Asmi, E.; Sellegri, K.; Depuy, R.; Venzac, H.; Villani, P.; Laj, P.; Aalto, P.; Ogren, J. A.; Swietlicki, E.; Williams, P.; Roldin, P.; Quincey, P.; Hüglin, C.; Fierz-Schmidhauser, R.; Gysel, M.; Weingartner, E.; Riccobono, F.; Santos, S.; Grüning, C.; Faloon, K.; Beddows, D.; Harrison, R.; Monahan, C.; Jennings, S. G.; O'Dowd, C. D.; Marinoni, A.; Horn, H. G.; Keck, L.; Jiang, J.; Scheckman, J.; McMurry, P. H.; Deng, Z.; Zhao, C. S.; Moerman, M.; Henzing, B.; de Leeuw, G.; Löschau, G.; Bastian, S. Atmos. Meas. Tech. 2012, $5(3), 657$.

[43] Vanhanen, J.; Mikkilä, J.; Lehtipalo, K.; Sipilä, M.; Manninen, H. E.; Siivola, E.; Petäjä, T.; Kulmala, M. Aerosol Sci. Technol. 2011, 45(4), 533.

[44] Hirsikko, A.; Nieminen, T.; Gagné, S.; Lehtipalo, K.; Manninen, H. E.; Ehn, M.; Hõrrak, U.; Kerminen, V. M.; Laakso, L.; McMurry, P. H.; Mirme, A.; Mirme, S.; Petäjä, T.; Tammet, H.; Vakkari, V.; Vana, M.; Kulmala, M. Atmos. Chem. Phys. 2011, 11(2), 767.

[45] Smith, J. N.; Dunn, M. J.; VanReken, T. M.; Iida, K.; Stolzenburg, M. R.; McMurry, P. H.; Huey, L. G. Geophys. Res. Lett. 2008, 35(4), L04808.

[46] Smith, J. N.; Moore, K. F.; McMurry, P. H.; Eisele, F. L. Aerosol Sci. Technol. 2004, 38(2), 100.

[47] Smith, J. N.; Moore, K. F.; Eisele, F. L.; Voisin, D.; Ghimire, A. K.; Sakurai, H.; McMurry, P. H. J. Geophys. Res.-Atmos. 2005, 110(D22), D22S03.

[48] Smith, J. N.; Barsanti, K. C.; Friedli, H. R.; Ehn, M.; Kulmala, M.; Collins, D. R.; Scheckman, J. H.; Williams, B. J.; McMurry, P. H. Proc. Natl. Acad. Sci. 2010, 107(15), 6634.

[49] Ross Pennington, M.; Johnston, M. V. Int. J. Mass Spectrom. 2012, 311, 64.

[50] Pennington, M. R.; Klems, J. P.; Bzdek, B. R.; Johnston, M. V.; J. Geophys. Res. 2012, 117, D00V10.

[51] Bzdek, B. R.; Zordan, C. A.; Luther, G. W.; Johnston, M. V.; Aerosol Sci. Technol. 2011, 45(8), 1041.

[52] Bzdek, B. R.; Zordan, C. A.; Pennington, M. R.; Luther, G. W.; Johnston, M. V. Environ. Sci. Technol. 2012, 46(8), 4365.

[53] Zhang, Q.; Stanier, C. O.; Canagaratna, M. R.; Jayne, J. T.; Worsnop, D. R.; Pandis, S. N.; Jimenez, J. L. Environ. Sci. Technol. 2004, 38(18), 4797.

[54] Sakurai, H.; Fink, M. A.; McMurry, P. H.; Mauldin, L.; Moore, K. F.; Smith, J. N.; Eisele, F. L. J. Geophys. Res. 2005, 110(D22), $\mathrm{D} 22 \mathrm{~S} 04$.

[55] Lee, S. H.; Allen, H. C. Anal. Chem. 2011.

[56] Wehner, B.; Siebert, H.; Stratmann, F.; Tuch, T.; Wiedensohler, A.; Petaja, T.; Dal Maso, M.; Kulmala, M. Tellus B 2007, 59(3), 362.
[57] Hussein, T.; Junninen, H.; Tunved, P.; Kristensson, A.; Dal Maso, M.; Riipinen, I.; Aalto, P. P.; Hansson, H. C.; Swietlicki, E.; Kulmala, M. Atmos. Chem. Phys. 2009, 9(14), 4699.

[58] Hussein, T.; Martikainen, J.; Junninen, H.; Sogacheva, L.; Wagner, R.; Dal Maso, M.; Riipinen, I.; Aalto, P. P.; Kulmala, M. Tellus B 2008, 60(4), 509.

[59] Vehkamäki, H.; Dal Maso, M.; Hussein, T.; Flanagan, R.; Hyvärinen, A.; Lauros, J.; Merikanto, P.; Mönkkönen, M.; Pihlatie, K.; Salminen, K.; Sogacheva, L.; Thum, T.; Ruuskanen, T. M.; Keronen, P.; Aalto, P. P.; Hari, P.; Lehtinen, K. E. J.; Rannik, Ü.; Kulmala, M. Atmos. Chem. Phys. 2004, 4, 2015.

[60] Wehner, B.; Wiedensohler, A. Atmos. Chem. Phys. 2003, 3, 867.

[61] Jeong, C. H.; Hopke, P. K.; Chalupa, D.; Utell, M. Environ. Sci. Technol. 2004, 38(7), 1933.

[62] Stanier, C. O.; Khlystov, A. Y.; Pandis, S. N. Atmos. Environ. 2004 $38(20), 3275$.

[63] Park, J.; Sakurai, H.; Vollmers, K.; McMurry, P. H. Atmos. Environ. 2004, 38(32), 5493.

[64] Komppula, M.; Sihto, S. L.; Korhonen, H.; Lihavainen, H.; Kerminen, V. M.; Kulmala, M.; Viisanen, Y. Atmos. Chem. Phys. 2006, 6, 2811.

[65] Hamed, A.; Joutsensaari, J.; Mikkonen, S.; Sogacheva, L.; Dal Maso, M.; Kulmala, M.; Cavalli, F.; Fuzzi, S.; Facchini, M. C.; Decesari, S.; Mircea, M.; Lehtinen, K. E. J.; Laaksonen, A. Atmos. Chem. Phys. 2007, 7, 355.

[66] Qian, S.; Sakurai, H.; McMurry, P. H. Atmos. Environ. 2007, 41(19), 4119.

[67] Dal Maso, M.; Hyvarinen, A.; Komppula, M.; Tunved, P.; Kerminen, V. M.; Lihavainen, H.; Viisanen, Y.; Hansson, H. C.; Kulmala, M. Tellus B 2008, 60(4), 495.

[68] Dal Maso, M.; Sogacheva, L.; Anisimov, M. P.; Arshinov, M.; Baklanov, A.; Belan, B.; Khodzher, T. V.; Obolkin, V. A.; Staroverova, A.; Vlasov, A.; Zagaynov, V. A.; Lushnikov, A.; Lyubovtseva, Y. S.; Riipinen, I.; Kerminen, V. M.; Kulmala, M. Boreal Environ. Res. 2008, 13(2), 81.

[69] Wehner, B.; Birmili, W.; Ditas, F.; Wu, Z.; Hu, M.; Liu, X.; Mao, J.; Sugimoto, N.; Wiedensohler, A. Atmos. Chem. Phys. 2008, 8(20), 6155 .

[70] Kivekas, N.; Sun, J.; Zhan, M.; Kerminen, V. M.; Hyvarinen, A.; Komppula, M.; Viisanen, Y.; Hong, N.; Zhang, Y.; Kulmala, M.; Zhang, X. C.; Deli, G.; Lihavainen, H. Atmos. Chem. Phys. 2009, $9(15), 5461$.

[71] Paasonen, P.; Sihto, S.-L.; Nieminen, T.; Vuollekoski, H.; Riipinen, I.; Plaß-Dülmer, C.; Berresheim, H.; Birmili, W.; Kulmala, M. Boreal Environ. Res. 2009, 14(4), 616.

[72] Shrestha, P.; Barros, A. P.; Khlystov, A. Atmos. Chem. Phys. 2010, 10(23), 11605.

[73] Spracklen, D. V.; Carslaw, K. S.; Merikanto, J.; Mann, G. W.; Reddington, C. L.; Pickering, S.; Ogren, J. A.; Andrews, E.; Baltensperger, U.; Weingartner, E.; Boy, M.; Kulmala, M.; Laakso, L.; Lihavainen, H.; Kivekas, N.; Komppula, M.; Mihalopoulos, N.; Kouvarakis, G.; Jennings, S. G.; O'Dowd, C.; Birmili, W.; Wiedensohler, A.; Weller, R.; Gras, J.; Laj, P.; Sellegri, K.; Bonn, B.; Krejci, R.; Laaksonen, A.; Hamed, A.; Minikin, A.; Harrison, R. M.; Talbot, R.; Sun, J. Atmos. Chem. Phys. 2010, 10(10), 4775.

[74] Shen, X. J.; Sun, J. Y.; Zhang, Y. M.; Wehner, B.; Nowak, A.; Tuch, T.; Zhang, X. C.; Wang, T. T.; Zhou, H. G.; Zhang, X. L.; Dong, F.; Birmili, W.; Wiedensohler, A. Atmos. Chem. Phys. 2011, 11(4), 1565 .

[75] Asmi, A.; Wiedensohler, A.; Laj, P.; Fjaeraa, A. M.; Sellegri, K.; Birmili, W.; Weingartner, E.; Baltensperger, U.; Zdimal, V.; Zikova, N.; Putaud, J. P.; Marinoni, A.; Tunved, P.; Hansson, H. C.; Fiebig, M.; Kivekäs, N.; Lihavainen, H.; Asmi, E.; Ulevicius, V.; Aalto, P. P.; Swietlicki, E.; Kristensson, A.; Mihalopoulos, N.; Kalivitis, N.; Kalapov, I.; Kiss, G.; de Leeuw, G.; Henzing, B.; Harrison, R. M.; Beddows, D.; O'Dowd, C.; Jennings, S. G.; Flentje, H.; Weinhold, K.; Meinhardt, F.; Ries, L.; Kulmala, M. Atmos. Chem. Phys. 2011, $11(11), 5505$.

[76] Charron, A.; Birmili, W.; Harrison, R. M. J. Geophys. Res. 2007, 112(D14), D14210.

[77] Suni, T.; Kulmala, M.; Hirsikko, A.; Bergman, T.; Laakso, L.; Aalto, P. P.; Leuning, R.; Cleugh, H.; Zegelin, S.; Hughes, D.; van Gorsel, E.; Kitchen, M.; Vana, M.; Hõrrak, U.; Mirme, S.; Mirme, A.; Sevanto, S.; Twining, J.; Tadros, C. Atmos. Chem. Phys. 2008, 8(1), 129.

[78] Vakkari, V.; Laakso, H.; Kulmala, M.; Laaksonen, A.; Mabaso, D.; 
Molefe, M.; Kgabi, N.; Laakso, L. Atmos. Chem. Phys. 2011, 11(7), 3333.

[79] Birmili, W.; Weinhold, K.; Nordmann, S.; Wiedensohler, A.; Spindler, G.; Mueller, K.; Herrmann, H.; Gnauk, T.; Pitz, M.; Cyrys, J.; Flentje, H.; Nickel, C.; Kuhlbusch, T. A. J.; Loschau, G.; Haase, D.; Meinhardt, F.; Schwerin, A.; Ries, L.; Wirtz, K. Gefahrst. Reinhalt. L. 2009, 69(4), 137.

[80] Wu, Z. J.; Hu, M.; Liu, S.; Wehner, B.; Bauer, S.; Ssling, A. M.; Wiedensohler, A.; Petaja, T.; Dal Maso, M.; Kulmala, M. J. Geophys. Res. 2007, 112(D9), D09209.

[81] Monkkonen, P.; Koponen, I. K.; Lehtinen, K. E. J.; Hameri, K.; Uma, R.; Kulmala, M. Atmos. Chem. Phys. 2005, 5, 57.

[82] Birmili, W.; Wiedensohler, A.; Plass-Dulmer, C.; Berresheim, H. Geophys. Res. Lett. 2000, 27(15), 2205.

[83] Dunn, M. J.; Jimenez, J. L.; Baumgardner, D.; Castro, T.; McMurry, P. H.; Smith, J. N. Geophys. Res. Lett. 2004, 31(10), L10102.

[84] Kulmala, M.; Petäjä, T.; Monkkonen, P.; Koponen, I. K.; Dal Maso, M.; Aalto, P. P.; Lehtinen, K. E. J.; Kerminen, V.-M. Atmos. Chem. Phys. 2005, 5, 409.

[85] O. Dowd, C. D.; Makela, J.; Vakeva, M.; Aalto, P.; de Leeuw, G.; Kunz, G. J.; Becker, E.; Hansson, H. C.; Allen, A. G.; Harrison, R. M.; Berresheim, H.; Geever, M.; Jennings, S. G.; Kulmala, M. J. Geophys. Res. 2002, 107(D19), 8107.

[86] Liu, S.; Hu, M.; Wu, Z. J.; Wehner, B.; Wiedensohler, A.; Cheng, Y. F. Atmos. Environ. 2008, 42(25), 6275.

[87] Held, A.; Nowak, A.; Birmili, W.; Wiedensohler, A.; Forkel, R.; Klemm, O. J. Geophys. Res. 2004, 109(D23), D23204.

[88] Asmi, E.; Frey, A.; Virkkula, A.; Ehn, M.; Manninen, H. E.; Timonen, H.; Tolonen-Kivimaki, O.; Aurela, M.; Hillamo, R.; Kulmala, M. Atmos. Chem. Phys. 2010, 10(9), 4253.

[89] Stanier, C. O.; Khlystov, A. Y.; Pandis, S. N. Aerosol Sci. Technol. 2004, 38, 253

[90] Riipinen, I.; Sihto, S.-L.; Kulmala, M.; Arnold, F.; Dal Maso, M.; Birmili, W.; Saarnio, K.; Teinilä, K.; Kerminen, V.-M.; Laaksonen, A.; Lehtinen, K. E. J. Atmos. Chem. Phys. 2007, 7(8), 1899.

[91] Yli-Juuti, T.; Riipinen, I.; Aalto, P. P.; Nieminen, T.; Maenhaut, W.; Janssens, I. A.; Claeys, M.; Salma, I.; Ocskay, R.; Hoffer, A.; Imre, K.; Kulmala, M. Boreal Environ. Res. 2009, 14(4), 683.

[92] Venzac, H.; Sellegri, K.; Laj, P.; Villani, P.; Bonasoni, P.; Marinoni, A.; Cristofanelli, P.; Calzolari, F.; Fuzzi, S.; Decesari, S.; Facchini, M. C.; Vuillermoz, E.; Verza, G. P. Proc. Natl. Acad. Sci. 2008, 105(41), 15666.

[93] Salma, I.; Borsós, T.; Weidinger, T.; Aalto, P.; Hussein, T.; Dal Maso, M.; Kulmala, M. Atmos. Chem. Phys. 2011, $11(3), 1339$.

[94] Cheung, H. C.; Morawska, L.; Ristovski, Z. D. Atmos. Chem. Phys. 2011, 11(8), 3823.

[95] Fuchs, N. A. The Mechanics of Aerosols, Pergamon, Oxford, 1964.

[96] Weber, R. J.; McMurry, P. H.; Mauldin, R. L.; Tanner, D. J.; Eisele, F. L.; Clarke, A. D.; Kapustin, V. N. Geophys. Res. Lett. 1999, 26(3), 307.

[97] Kulmala, M.; Laaksonen, A.; Pirjola, L. J. Geophys. Res.-Atmos. 1998, 103(D7), 8301

[98] Wilemski, G. J. Chem. Phys. 1984, 80(3), 1370.

[99] Napari, I.; Kulmala, M.; Vehkamäki, H. J. Chem. Phys. 2002, $117(18), 8418$

[100] Korhonen, P.; Kulmala, M.; Laaksonen, A.; Viisanen, Y.; McGraw, R.; Seinfeld, J. H. J. Geophys. Res. 1999, 104(D21), 26349.

[101] Scott, W. D.; Cattell, F. C. R. Atmos. Environ. 1979, 13(2), 307.

[102] Napari, I.; Noppel, M.; Vehkamäki, H.; Kulmala, M. J. Chem. Phys. 2002, 116(10), 4221.

[103] Yu, F. Q.; Turco, R. P. Geophys. Res. Lett. 1997, 24(15), 1927.

[104] Yu, F. Q.; Turco, R. Atmos. Chem. Phys. 2008, 8(20), 6085.

[105] Jimenez, J. L.; Bahreini, R.; Cocker, D. R.; Zhuang, H.; Varutbangkul, V.; Flagan, R. C.; Seinfeld, J. H.; O'Dowd, C. D.; Hoffmann, T. J. Geophys. Res. 2003, 108(D10), 4318.

[106] O'Dowd, C. D.; Jimenez, J. L.; Bahreini, R.; Flagan, R. C.; Seinfeld, J. H.; Hameri, K.; Pirjola, L.; Kulmala, M.; Jennings, S. G.; Hoffmann, T. Nature 2002, 417(6889), 632.

[107] de Leeuw, G.; Kunz, G. J.; Buzorius, G.; O'Dowd, C. J. Geophys. Res. 2002, 107(D19), 8102.

[108] Vuollekoski, H.; Kerminen, V.-M.; Anttila, T.; Sihto, S.-L.; Vana, M.; Ehn, M.; Korhonen, H.; McFiggans, G.; O'Dowd, C. D.; Kulmala, M. J. Geophys. Res.- Atmos. 2009, 114(D2), D02206.

[109] Kulmala, M.; Pirjola, U.; Makela, J. M. Nature 2000, 404(6773), 66.

[110] Kulmala, M.; Lehtinen, K. E. J.; Laaksonen, A. Atmos. Chem. Phys.
2006, 6, 787 .

[111] Sihto, S.-L.; Kulmala, M.; Kerminen, V.-M.; Dal Maso, M.; Petäjä, T.; Riipinen, I.; Korhonen, H.; Arnold, F.; Janson, R.; Boy, M.; Laaksonen, A.; Lehtinen, K. E. J. Atmos. Chem. Phys. 2006, 6, 4079.

[112] Nieminen, T.; Manninen, H. E.; Sihto, S.-L.; Yli-Juuti, T.; Mauldin III, R. L.; Petäjä, T.; Riipinen, I.; Kerminen, V.-M.; Kulmala, M. Environ. Sci. Technol. 2009, 43(13), 4715.

[113] Kuang, C.; McMurry, P. H.; McCormick, A. V.; Eisele, F. L. J. Geophys. Res. 2008, 113(D10), D10209.

[114] Boy, M.; Karl, T.; Turnipseed, A.; Mauldin, R. L.; Kosciuch, E.; Greenberg, J.; Rathbone, J.; Smith, J.; Held, A.; Barsanti, K.; Wehner, B.; Bauer, S.; Wiedensohler, A.; Bonn, B.; Kulmala, M.; Guenther, A. Atmos. Chem. Phys. 2008, 8(6), 1577.

[115] Wang, Z. B.; Hu, M.; Yue, D. L.; Zheng, J.; Zhang, R. Y.; Wiedensohler, A.; Wu, Z. J.; Nieminen, T.; Boy, M. Atmos. Chem. Phys. 2011, 11(24), 12663.

[116] Chen, M.; Titcombe, M.; Jiang, J.; Jen, C.; Kuang, C.; Fischer, M. L.; Eisele, F. L.; Siepmann, J. I.; Hanson, D. R.; Zhao, J.; McMurry, P. H. Proc. Natl. Acad. Sci. 2012, 109(46), 18713.

[117] Dawson, M. L.; Varner, M. E.; Perraud, V.; Ezell, M. J.; Gerber, R. B.; Finlayson-Pitts, B. J. Proc. Natl. Acad. Sci. 2012, 109(46), 18719.

[118] Weber, R. J.; Mcmurry, P. H.; Eisele, F. L.; Tanner, D. J. J. Atmos. Sci. 1995, 52(12), 2242

[119] Weber, R. J.; Marti, J. J.; McMurry, P. H.; Eisele, F. L.; Tanner, D. J.; Jefferson, A. J. Geophys. Res.- Atmos. 1997, 102 (D4), 4375.

[120] Weber, R. J.; Mcmurry, P. H.; Mauldin, L.; Tanner, D. J.; Eisele, F. L.; Brechtel, F. J.; Kreidenweis, S. M.; Kok, G. L.; Schillawski, R. D.; Baumgardner, D. J. Geophys. Res. 1998, 103(D13), 16385.

[121] McMurry, P. H.; Fink, M.; Sakurai, H.; Stolzenburg, M. R.; Mauldin, R. L.; Smith, J.; Eisele, F.; Moore, K.; Sjostedt, S.; Tanner, D.; Huey, L. G.; Nowak, J. B.; Edgerton, E.; Voisin, D. J. Geophys. Res. 2005, 110(D22), D22S02.

[122] Iida, K.; Stolzenburg, M. R.; McMurry, P. H.; Smith, J. N. J. Geophys. Res. 2008, 113(D5), D05207.

[123] Fiedler, V.; Dal Maso, M.; Boy, M.; Aufmhoff, H.; Hoffmann, J.; Schuck, T.; Birmili, W.; Hanke, M.; Uecker, J.; Arnold, F.; Kulmala, M. Atmos. Chem. Phys. 2005, 5, 1773.

[124] Boy, M.; Rannik, U.; Lehtinen, K. E. J.; Tarvainen, V.; Hakola, H.; Kulmala, M. J. Geophys. Res.; 2003, 108(D21), 4667.

[125] Boy, M.; Kulmala, M.; Ruuskanen, T. M.; Pihlatie, M.; Reissell, A.; Aalto, P. P.; Keronen, P.; Dal Maso, M.; Hellen, H.; Hakola, H.; Jansson, R.; Hanke, M.; Arnold, F. Atmos. Chem. Phys. 2005, 5, 863.

[126] Kuang, C.; Riipinen, I.; Sihto, S. L.; Kulmala, M.; McCormick, A V.; McMurry, P. H. Atmos. Chem. Phys. 2010, 10(17), 8469.

[127] Paasonen, P.; Nieminen, T.; Asmi, E.; Manninen, H. E.; Petäjä, T.; Plass-Dülmer, C.; Flentje, H.; Birmili, W.; Wiedensohler, A.; Hõrrak, U.; Metzger, A.; Hamed, A.; Laaksonen, A.; Facchini, M. C.; Kerminen, V.-M.; Kulmala, M. Atmos. Chem. Phys. 2010, $10(22), 11223$

[128] Gaydos, T. M.; Stanier, C. O.; Pandis, S. N. J. Geophys. Res. 2005 110(D7), D07S12.

[129] Kerminen, V.-M.; Petäjä, T.; Manninen, H. E.; Paasonen, P.; Nieminen, T.; Sipilä, M.; Junninen, H.; Ehn, M.; Gagné, S.; Laakso, L.; Riipinen, I.; Vehkamäki, H.; Kurten, T.; Ortega, I. K.; Dal Maso, M.; Brus, D.; Hyvärinen, A.; Lihavainen, H.; Leppä, J.; Lehtinen, K. E. J.; Mirme, A.; Mirme, S.; Hõrrak, U.; Berndt, T.; Stratmann, F.; Birmili, W.; Wiedensohler, A.; Metzger, A.; Dommen, J.; Baltensperger, U.; Kiendler-Scharr, A.; Mentel, T. F.; Wildt, J.; Winkler, P. M.; Wagner, P. E.; Petzold, A.; Minikin, A.; Plass-Dülmer, C.; Pöschl, U.; Laaksonen, A.; Kulmala, M. Atmos. Chem. Phys. 2010, 10(22), 10829.

[130] Berndt, T.; Stratmann, F.; Sipilä, M.; Vanhanen, J.; Petäjä, T.; Mikkilä, J.; Grüner, A.; Spindler, G.; Lee Mauldin Iii, R.; Curtius, J.; Kulmala, M.; Heintzenberg, J. Atmos. Chem. Phys. 2010, 10(15), 7101

[131] Kirkby, J.; Curtius, J.; Almeida, J.; Dunne, E.; Duplissy, J.; Ehrhart, S.; Franchin, A.; Gagne, S.; Ickes, L.; Kurten, A.; Kupc, A.; Metzger, A.; Riccobono, F.; Rondo, L.; Schobesberger, S.; Tsagkogeorgas, G.; Wimmer, D.; Amorim, A.; Bianchi, F.; Breitenlechner, M.; David, A.; Dommen, J.; Downard, A.; Ehn, M.; Flagan, R. C.; Haider, S.; Hansel, A.; Hauser, D.; Jud, W.; Junninen, H.; Kreissl, F.; Kvashin, A.; Laaksonen, A.; Lehtipalo, K.; Lima, J.; Lovejoy, E. R.; Makhmutov, V.; Mathot, S.; Mikkila, J.; Minginette, 
P.; Mogo, S.; Nieminen, T.; Onnela, A.; Pereira, P.; Petaja, T.; Schnitzhofer, R.; Seinfeld, J. H.; Sipila, M.; Stozhkov, Y.; Stratmann, F.; Tome, A.; Vanhanen, J.; Viisanen, Y.; Vrtala, A.; Wagner, P. E.; Walther, H.; Weingartner, E.; Wex, H.; Winkler, P. M.; Carslaw, K. S.; Worsnop, D. R.; Baltensperger, U.; Kulmala, M. Nature 2011, 476(7361), 429.

[132] Jimenez, J. L.; Canagaratna, M. R.; Donahue, N. M.; Prevot, A. S. H.; Zhang, Q.; Kroll, J. H.; DeCarlo, P. F.; Allan, J. D.; Coe, H.; Ng, N. L.; Aiken, A. C.; Docherty, K. S.; Ulbrich, I. M.; Grieshop, A. P.; Robinson, A. L.; Duplissy, J.; Smith, J. D.; Wilson, K. R.; Lanz, V. A.; Hueglin, C.; Sun, Y. L.; Tian, J.; Laaksonen, A.; Raatikainen, T.; Rautiainen, J.; Vaattovaara, P.; Ehn, M.; Kulmala, M.; Tomlinson, J. M.; Collins, D. R.; Cubison, M. J.; Dunlea, E. J.; Huffman, J. A.; Onasch, T. B.; Alfarra, M. R.; Williams, P. I.; Bower, K.; Kondo, Y.; Schneider, J.; Drewnick, F.; Borrmann, S.; Weimer, S.; Demerjian, K.; Salcedo, D.; Cottrell, L.; Griffin, R.; Takami, A.; Miyoshi, T.; Hatakeyama, S.; Shimono, A.; Sun, J. Y.; Zhang, Y. M.; Dzepina, K.; Kimmel, J. R.; Sueper, D.; Jayne, J. T.; Herndon, S. C.; Trimborn, A. M.; Williams, L. R.; Wood, E. C.; Middlebrook, A. M.; Kolb, C. E.; Baltensperger, U.; Worsnop, D. R. Science 2009, 326(5959), 1525.

[133] Kavouras, I. G.; Mihalopoulos, N.; Stephanou, E. G. Nature 1998, 395(6703), 683

[134] O. Dowd, C. D.; Aalto, P.; Hameri, K.; Kulmala, M.; Hoffmann, T. Nature 2002, 416(6880), 497.

[135] Makela, J. M.; Yli-Koivisto, S.; Hiltunen, V.; Seidl, W.; Swietlicki, E.; Teinila, K.; Sillanpaa, M.; Koponen, I. K.; Paatero, J.; Rosman, K.; Hameri, K. Tellus B 2001, 53(4), 380.

[136] Zhang, R. Y.; Suh, I.; Zhao, J.; Zhang, D.; Fortner, E. C.; Tie, X. X.; Molina, L. T.; Molina, M. J. Science 2004, 304(5676), 1487.

[137] Barsanti, K. C.; McMurry, P. H.; Smith, J. N. Atmos. Chem. Phys. 2009, 9(9), 2949.

[138] Erupe, M. E.; Viggiano, A. A.; Lee, S. H. Atmos. Chem. Phys. 2011, 11(10), 4767.

[139] Wang, L.; Lal, V.; Khalizov, A. F.; Zhang, R. Y. Environ. Sci. Technol. 2010, 44(7), 2461.

[140] Metzger, A.; Verheggen, B.; Dommen, J.; Duplissy, J.; Prevot, A. S. H.; Weingartner, E.; Riipinen, I.; Kulmala, M.; Spracklen, D. V.; Carslaw, K. S.; Baltensperger, U. Proc. Natl. Acad. Sci. 2010, 107(15), 6646.

[141] Laaksonen, A.; Kulmala, M.; O'Dowd, C. D.; Joutsensaari, J.; Vaattovaara, P.; Mikkonen, S.; Lehtinen, K. E. J.; Sogacheva, L.; Dal Maso, M.; Aalto, P.; Petäjä, T.; Sogachev, A.; Yoon, Y. J.; Lihavainen, H.; Nilsson, D.; Facchini, M. C.; Cavalli, F.; Fuzzi, S.; Hoffmann, T.; Arnold, F.; Hanke, M.; Sellegri, K.; Umann, B.; Junkermann, W.; Coe, H.; Allan, J. D.; Alfarra, M. R.; Worsnop, D. R.; Riekkola, M. L.; Hyötyläinen, T.; Viisanen, Y. Atmos. Chem. Phys. 2008, 8(10), 2657.

[142] Wang, L.; Khalizov, A. F.; Zheng, J.; Xu, W.; Ma, Y.; Lal, V.; Zhang, R. Y. Nat. Geosci. 2010, 3(4), 238.

[143] Kurtén, T.; Loukonen, V.; Vehkamäki, H.; Kulmala, M. Atmos. Chem. Phys. 2008, 8(14), 4095.

[144] Qiu, C.; Wang, L.; Lal, V.; Khalizov, A. F.; Zhang, R. Environ. Sci. Technol. 2011, 45(11), 4748.
[145] Bzdek, B. R.; Ridge, D. P.; Johnston, M. V. Atmos. Chem. Phys. 2010, $10(8), 3495$.

[146] Bzdek, B. R.; Ridge, D. P.; Johnston, M. V. J. Phys. Chem. A 2010 114(43), 11638

[147] Bzdek, B. R.; Ridge, D. P.; Johnston, M. V. Atmos. Chem. Phys. 2011, 11(16), 8735 .

[148] Kiendler-Scharr, A.; Wildt, J.; Dal Maso, M.; Hohaus, T.; Kleist, E.; Mentel, T. F.; Tillmann, R.; Uerlings, R.; Schurr, U.; Wahner, A. Nature 2009, 461(7262), 381.

[149] Kanawade, V. P.; Jobson, B. T.; Guenther, A. B.; Erupe, M. E.; Pressley, S. N.; Tripathi, S. N.; Lee, S. H. Atmos. Chem. Phys. 2011, 11(12), 6013.

[150] Donahue, N. M.; Trump, E. R.; Pierce, J. R.; Riipinen, I. Geophys. Res. Lett. 2011, 38(16), L16801.

[151] Wang, L.; Xu, W.; Khalizov, A. F.; Zheng, J.; Qiu, C.; Zhang, R. Y. J. Phys. Chem. A 2011, 115(32), 8940.

[152] Wehner, B.; Wiedensohler, A.; Tuch, T. M.; Wu, Z. J.; Hu, M.; Slanina, J.; Kiang, C. S. Geophys. Res. Lett. 2004, 31(22), L22108.

[153] Du, J.; Cheng, T.; Zhang, M.; Chen, J.; He, Q.; Wang, X.; Zhang, R.; Tao, J.; Huang, G.; Li, X.; Zha, S. Aerosol Air Qual. Res. 2012, 12(6), 1362.

[154] Gao, J.; Chai, F.; Wang, T.; Wang, S.; Wang, W. J. Environ. Sci. 2012, 24(1), 14

[155] Gao, J.; Chai, F.; Wang, T.; Wang, W. Particuology 2011, 9(6), 611.

[156] Gao, J.; Wang, T.; Zhou, X.; Wu, W.; Wang, W. Atmos. Environ. 2009, 43(4), 829.

[157] Yue, D. L.; Hu, M.; Zhang, R. Y.; Wang, Z. B.; Zheng, J.; Wu, Z. J.; Wiedensohler, A.; He, L. Y.; Huang, X. F.; Zhu, T. Atmos. Chem. Phys. 2010, 10(10), 4953.

[158] Zhang, Y. M.; Zhang, X. Y.; Sun, J. Y.; Lin, W. L.; Gong, S. L.; Shen, X. J.; Yang, S. Tellus B 2011, 63(3), 382.

[159] Wiedensohler, A.; Cheng, Y. F.; Nowak, A.; Wehner, B.; Achtert, P.; Berghof, M.; Birmili, W.; Wu, Z. J.; Hu, M.; Zhu, T.; Takegawa, N.; Kita, K.; Kondo, Y.; Lou, S. R.; Hofzumahaus, A.; Holland, F.; Wahner, A.; Gunthe, S. S.; Rose, D.; Su, H.; Pöschl, U. J. Geophys. Res.-Atmos. 2009, 114(D2), D00G08.

[160] Yue, D. L.; Hu, M.; Wu, Z. J.; Wang, Z. B.; Guo, S.; Wehner, B.; Nowak, A.; Achtert, P.; Wiedensohler, A.; Jung, J. S.; Kim, Y. J.; Liu, S. C. J. Geophys. Res. 2009, 114(D2), D00G12.

[161] Herrmann, E.; Ding, A. J.; Petäjä, T.; Yang, X. Q.; Sun, J. N.; Qi, X. M.; Manninen, H.; Hakala, J.; Nieminen, T.; Aalto, P. P.; Kerminen, V. M.; Kulmala, M.; Fu, C. B. Atmos. Chem. Phys. Discuss. 2013, 13(1), 1455.

[162] Lin, P.; Hu, M.; Wu, Z.; Niu, Y.; Zhu, T. Atmos. Environ. 2007, 41(32), 6784.

[163] Gong, Y. G.; Hu, M.; Cheng, Y.; Su, H.; Yue, D.; Liu, F.; Wiedensohler, A.; Wang, Z.; Kalesse, H.; Liu, S.; Wu, Z.; Xiao, K.; Mi, P.; Zhang, Y. Atmos. Environ. 2010, 44(27), 3278.

[164] Li, W. J.; Zhang, D. Z.; Shao, L. Y.; Zhou, S. Z.; Wang, W. X. Atmos. Chem. Phys. 2011, 11(22), 11733.

[165] Guo, H.; Wang, D. W.; Cheung, K.; Ling, Z. H.; Chan, C. K.; Yao, X. H. Atmos. Chem. Phys. 2012, 12(20), 9923.

[166] Gong, Y. G.; Su, H.; Cheng, Y.; Liu, F.; Wu, Z.; Hu, M.; Zeng, L.; Zhang, Y. Adv. Atmos. Sci. 2008, 25(3), 427. 